\title{
Fiber-Optic Microsphere-Based Arrays for Multiplexed Biological Warfare Agent Detection
}

\author{
Linan Song, Soohyoun Ahn, and David R. Walt ${ }^{*}$ \\ Department of Chemistry, Tufts University, Medford, Massachusetts 02155
}

Table of content:

1. Gel analysis of the asymmetric PCR amplifications of target BWAs from the autoclaved bacterial cultures with their specific primer pairs.

* Corresponding author Tel.: +1-617-6273470; fax: +1-627-6273443

Email address: david.walt@tufts.edu 
Supporting information:

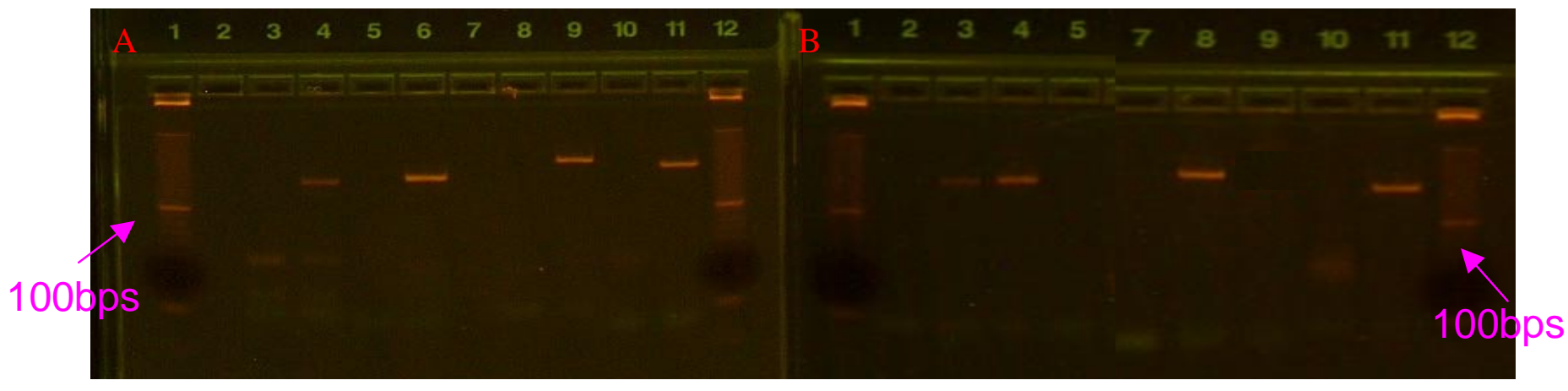

\begin{tabular}{llllllllllll|lllllllllllll}
1 & 2 & 3 & 4 & 5 & 6 & 7 & 8 & 9 & 10 & il & 12 & $D$ & 1 & 2 & 3 & 4 & 5 & 6 & 7 & 8 & 9 & 10 & 11 & 12
\end{tabular}

-

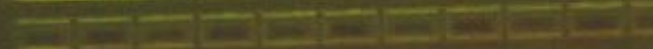

-

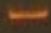

$100 \mathrm{bps}$

Figure S-1 Gel analysis of the asymmetric PCR amplifications of target BWAs from the autoclaved bacterial cultures with their specific primer pairs. The asymmetric PCRs were run with only one primer pair as described in the experimental section (see reference 35 for Primer sequences information). A. Amplification of Bacillus thuringiensis kurstaki (BTK) and Vaccinia virus (VA): lane 1, DNA ladder; lane 2, blank; lane 3, BTK1-c; lane 4, BTK1-s; lane 5, BTK2-c; lane 6, BTK2-s; lane 7, blank; lane 8, VA1-c; lane 9, VA1-s; lane 10, VA2-c; lane 11, VA2-s; and lane 12, DNA ladder. B. Amplification of Francisella tularensis (FT) and Yersinia pestis (YP): lane 1, DNA ladder; lane 2, FT1-c; lane 3, FT1-s; lane 4, FT2-s; lane 5, FT2-c, lane 7, YP1-c; lane 8, YP1-s; lane 9, blank; lane 10, YP2-c; lane 11, YP2-s, and lane 12, DNA ladder. C. Amplification of Bacillus anthracis (BA): lane 1, BA1-s; lane 2, BA2-c; lane 3, BA2-s; lane 4, BA3-c; lane 5, BA3-s; lane 6, BA4-c; lane 7, BA4-s; lane 8, BA5-c; lane 9, BA5-s; lane 10, BA6-c; lane 11, BA6-s, and lane 12, DNA ladder. D. Amplification of Clostridium botulinum (CB): lane 1, CB1-c; lane 2, CB1-s; lane 3, CB2-c; lane 4, CB2-s, lane 5-11, blank; and lane 12, DNA ladder. All the PCR controls were run with no template. 
c: negative control; s: spent media of the autoclaved bacterial cultures samples for the target BWAs of interest. 